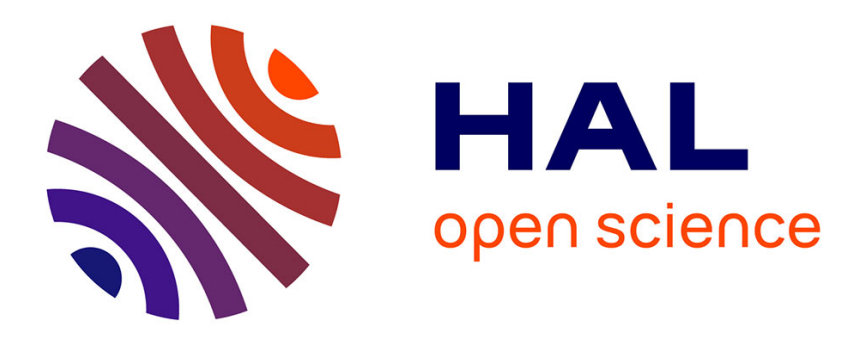

\title{
Effect of thermal noise and initial conditions in the dynamics of a mean-field ferromagnet
}

O. Golinelli, B. Derrida

\section{To cite this version:}

O. Golinelli, B. Derrida. Effect of thermal noise and initial conditions in the dynamics of a mean-field ferromagnet. Journal de Physique, 1988, 49 (10), pp.1663-1671. 10.1051/jphys:0198800490100166300 — jpa-00210847

\section{HAL Id: jpa-00210847 https://hal.science/jpa-00210847}

Submitted on 1 Jan 1988

HAL is a multi-disciplinary open access archive for the deposit and dissemination of scientific research documents, whether they are published or not. The documents may come from teaching and research institutions in France or abroad, or from public or private research centers.
L'archive ouverte pluridisciplinaire HAL, est destinée au dépôt et à la diffusion de documents scientifiques de niveau recherche, publiés ou non, émanant des établissements d'enseignement et de recherche français ou étrangers, des laboratoires publics ou privés. 


\title{
Effect of thermal noise and initial conditions in the dynamics of a mean- field ferromagnet
}

\author{
O. Golinelli and B. Derrida \\ Service de Physique Théorique (*) de Saclay, F-91191 Gif-sur-Yvette Cedex, France
}

(Reçu le 18 mai 1988, accepté le 16 juin 1988)

\begin{abstract}
Résumé. - Pour un ferromagnétique en champ moyen, nous calculons en fonction de la température la probabilité que deux configurations soumises au même bruit thermique tombent dans des vallées opposées. Pour un système de $N$ spins, nous montrons que le temps pour que deux configurations se rejoignent augmente commme $\log N$ quand les deux configurations se trouvent dans la même vallée et comme une exponentielle de $N$ quand elles appartiennent à des vallées différentes. La pente de cette croissance exponentielle donne une mesure de la hauteur de barrière.

Abstract. - For a mean field ferromagnet, we calculate as a function of temperature the probability that two configurations submitted to the same thermal noise fall into opposite valleys. For a system of $N$ spins, we show that the time for two configurations to meet increases like $\log N$ when the two configurations belong to the same valley and exponentially with $N$ when the two configurations belong to different valleys. The slope of this exponential growth gives a measure of the barrier height.
\end{abstract}

\section{Introduction.}

It has been recently suggested [1] that looking at the time evolution of two or more copies of a same system subjected to the same thermal noise could be a way of studying phase transitions from a dynamical point of view. The main idea is that in a high temperature phase, two different initial configurations end up by becoming identical whereas in a low temperature phase, they have a finite probability of falling into different valleys and thus of remaining different after a long time (which would be infinite for an infinite system). Monte Carlo calculations [1] done for the $3 \mathrm{~d}$ Ising ferromagnet have shown that the probability $P(t)$ that two different initial conditions have not met at time $t$ does not vanish for long times $t$ only below the critical temperature $T_{\mathrm{c}}$ of the ferromagnet. In the case of $3 \mathrm{~d}$ spin glasses [1], a dynamical phase transition (below which $P(t) \neq 0$ for long times) was found at a temperature much higher than what is usually believed to be the spin glass transition. Other Monte

(*) Laboratoire de l'Institut de Recherche Fondamentale du Commissariat à l'Energie Atomique
Carlo simulations using again the comparison of two configurations submitted to the same thermal noise give for the 2D ANNNI model clear dynamical phase transitions close to the phase boundaries of the ferromagnetic, antiphase and floating phases [2].

Since this method of comparing the time evolution of configurations submitted to the same thermal noise has not been yet used in many situations, it is useful, as a test, to see what it would give for the simplest systems. In the present paper, we consider the case of a mean field ferromagnet (the infinite range Ising model). The system is defined as $N$ Ising spins which interact through the following hamiltonian $\mathscr{H}$

$$
\mathfrak{H}=-\sum_{i<j} J_{i j} S_{i} S_{j}
$$

By definition of the model, all the interactions $J_{i j}$ are equal

$$
J_{i j}=J / N \quad \text { with } \quad J=1 .
$$

(In the infinite range model, one has to scale the strength of the interactions with $N$ in order to keep an extensive free energy). 
We will mostly consider the following parallel dynamics : if the system is in a configuration $\left\{S_{i}(t)\right\}$ at time $t$, it is updated according to the following rules:

(1) One computes the $N$ probabilities $p_{i}(t)$

$$
p_{i}(t)=\frac{1}{2}+\frac{1}{2} \tanh \left(\frac{1}{T} \sum_{j \neq i} J_{i j} S_{j}(t)\right)
$$

where $T$ is the temperature.

(2) One updates the $N$ spins according to

$$
\begin{aligned}
S_{i}(t) & =1 \text { with probability } p_{i}(t) \\
& =-1 \text { with probability } 1-p_{i}(t) .
\end{aligned}
$$

In practice, one chooses $N$ random numbers $z_{i}(t)$ uniformly distributed between 0 and 1 and one computes the new configuration $\left\{S_{i}(t+1)\right\}$ by

$$
S_{i}(t+1)=\operatorname{sign}\left[p_{i}(t)-z_{i}(t)\right] .
$$

These dynamics are parallel since all the spins are updated simultaneously. In that case, one can show that the probability $\mathfrak{T}_{t}\left(\left\{S_{i}\right\}\right)$ of finding the system in a configuration $\left\{S_{i}\right\}$ at time $t$ converges in the long time limit to an equilibrium $\mathfrak{T}_{\mathrm{eq}}\left(\left\{S_{i}\right\}\right)$ which is given by [3]

$$
\mathfrak{T}_{\mathrm{eq}}\left(\left\{S_{i}\right\}\right)=\mathrm{cst} \prod_{i=1}^{N} \cosh \left(\sum_{j} \frac{J_{i j} S_{j}}{T}\right) .
$$

This equilibrium probability distribution is different from the usual Boltzmann distribution

$$
\tilde{\mathfrak{T}}_{\text {eq }}\left(\left\{S_{i}\right\}\right)=\operatorname{cst} \exp \left[\sum_{i<j} \frac{J_{i j} S_{i} S_{j}}{T}\right]
$$

which would be the equilibrium distribution for sequential updating (for which during each time interval $\Delta t=\frac{1}{N}$, one spin $i$ is chosen at random and is updated according to (3) and (5)). However distributions (6) and (7) have usually very similar properties [4]. For example in the infinite range case (2), they both have a Curie temperature at

$$
T_{\mathrm{c}} / J=1
$$

and for $T<T_{\mathrm{c}}$ the same spontaneous magnetization given by

$$
m=\tanh \left(\frac{J}{T} m\right) .
$$

In the present paper, we want to compare the time evolution of two configurations submitted to the same thermal noise. This means that we choose two different initial configurations $\left\{S_{i}(0)\right\}$ and $\left\{\tilde{S}_{i}(0)\right\}$ and we make them evolve according to formulas (3) and (5) with the condition that at each time step, the same numbers $\left\{z_{i}(t)\right\}$ are used to update both configurations. Therefore

$$
\begin{aligned}
& S_{i}(t+1)=\operatorname{sign} \times \\
& \quad \times\left[\frac{1}{2}+\frac{1}{2} \tanh \left(\frac{1}{T} \sum_{j} J_{i j} S_{j}(t)\right)-z_{i}(t)\right] \\
& \tilde{S}_{i}(t+1)=\operatorname{sign} \times \\
& \quad \times\left[\frac{1}{2}+\frac{1}{2} \tanh \left(\frac{1}{T} \sum_{j} J_{i j} \tilde{S}_{j}(t)\right)-z_{i}(t)\right] .
\end{aligned}
$$

When one compares two configurations submitted to the same thermal noise, there are two simple quantities which can be considered. The survival probability $P(t)$ which is the probability that the two configurations are still different at time $t$ and the average distance $d(t)$ between two configurations defined by

$$
d(t)=\frac{1}{4 N} \sum_{i=1}^{N}\left(S_{i}(t)-\tilde{S}_{i}(t)\right)^{2} .
$$

Of course if two configurations become identical at a certain time $t$, they remain identical at any later time. The main goal of the present paper is to calculate the probability $P(t)$ when $t$ becomes large. Of course $1-P(t)$ can be interpreted as the probability that two initial configurations fall into the same valley.

In section 2 , we calculate the probability distribution of the magnetisation and of the distance between two initial configurations after a finite number of time steps. It is during these first time steps of the dynamics that the combined effects of the initial condition and of the thermal noise make each configuration choose what valley it will fall into.

In section 3, we show that once two configurations are in the same valley, it takes them a typical time of order $\log N$ to become identical.

In section 4 , we present some numerical simulations showing finite size effects in the low temperature phase. For a finite system of $N$ spins, two configurations always end up by becoming identical. We measure the time it takes for two configurations to meet and we see that this time increases with $N$ like the typical time for a configuration to go from one phase to the other.

\section{Dynamics after a finite number of time steps.}

In this section, we are going to compare the time evolution of two different configurations submitted to the same thermal noise (i.e. updated with the same random numbers $z_{i}(t)$ in Eq. (10)). As explained in the appendix (see the paragraph after Eq. (A.11)), the calculation is valid for $N \rightarrow \infty$ and for $t$ finite. 
We will consider two situations which had been studied in the numerical simulations of the 3 dimensional ferromagnet [1] :

(1) The configuration $\left\{S_{i}(0)\right\}$ is chosen at random and the configuration $\left\{\tilde{S}_{i}(0)\right\}$ is opposite to it, so that $d(0)=1$.

(2) The two configurations $\left\{S_{i}(0)\right\}$ and $\left\{\tilde{S}_{i}(0)\right\}$ are random so that $d(0)=\frac{1}{2}+O\left(N^{-1 / 2}\right)$.

Because the system is an infinite ranged model (see Eq. (1)), the two configurations can be described by 4 numbers $n_{1}, n_{2}, n_{3}$ and $n_{4}: n_{1}$ is the number of spins $i$ such that $S_{i}=\tilde{S}_{i}=+1, n_{2}$ such that $S_{i}=-\tilde{S}_{i}=+1, n_{3}$ such that $S_{i}=-\tilde{S}_{i}=-1$, $n_{4}$ such that $S_{i}=\tilde{S}_{i}=-1$. Of course one always has

$$
n_{1}+n_{2}+n_{3}+n_{4}=N
$$

and the total magnetizations $M_{1}$ and $M_{2}$ are given by

$$
\begin{aligned}
& M_{1}=n_{1}+n_{2}-n_{3}-n_{4} \\
& M_{2}=n_{1}-n_{2}+n_{3}-n_{4} .
\end{aligned}
$$

For the initial condition (1), $n_{1}=n_{4}=0$ and the probability distribution of $n_{2}$ and $n_{3}$ is

$$
P^{(1)}\left(n_{2}, n_{3}\right)=\frac{N !}{n_{2} ! n_{3} !} \times \frac{1}{2^{N}}
$$

whereas for the initial condition (2), each site has a probability $1 / 4$ of contributing to $n_{1}, n_{2}, n_{3}$ or $n_{4}$ and therefore

$$
P^{(2)}\left(n_{1}, n_{2}, n_{3}, n_{4}\right)=\frac{N !}{n_{1} ! n_{2} ! n_{3} ! n_{4} !} \times \frac{1}{4^{N}} .
$$

Because the two configurations $\left\{S_{i}\right\}$ and $\left\{\tilde{S}_{i}\right\}$ play symmetric roles, one can always choose $M_{1} \geq M_{2}$ so that $n_{2} \geq n_{3}$. Moreover since the probability that $n_{2}=n_{3}$ vanishes as $N \rightarrow \infty$, one can always neglect these events when $N$ is large. Therefore the condition

$$
n_{2} \geq n_{3}+1
$$

is satisfied (which gives condition (A.1) of the appendix) with probability one in the initial condition and the distributions (14) and (15) become

$$
\begin{aligned}
& P^{(1)}\left(n_{2}, n_{3}\right)=2 \frac{N !}{n_{2} ! n_{3} !} \frac{1}{2^{N}} \theta\left(n_{2}-n_{3}-1\right) \\
& P^{(2)}\left(n_{1}, n_{2}, n_{3}, n_{4}\right)= \\
& \quad=2 \frac{N !}{n_{1} ! n_{2} ! n_{3} ! n_{4} !} \frac{1}{4^{N}} \theta\left(n_{2}-n_{3}-1\right) .
\end{aligned}
$$

The probability $Q_{0}\left(M_{1}, M_{2}\right)$ of $M_{1}$ and $M_{2}$ can then be obtained from (17) and (18)

$$
\begin{aligned}
& Q_{0}\left(M_{1}, M_{2}\right)=\frac{1}{\pi N} \sqrt{A_{0} B_{0}} \times \\
& \quad \times \exp \left[-\frac{A_{0}\left(M_{1}+M_{2}\right)^{2}+B_{0}\left(M_{1}-M_{2}\right)^{2}}{4 N}\right] \\
& \quad \times \theta\left(M_{1}-M_{2}\right)
\end{aligned}
$$

where $A_{0}$ and $B_{0}$ are given by

$$
A_{0}=\infty \quad B_{0}=1 / 2
$$

for the initial condition (1) (i.e. for two opposite initial configurations) whereas

$$
A_{0}=1 \text { and } B_{0}=1
$$

for the initial condition (2).

In the appendix, we have shown (A.16), (A.18) that if the probability distribution of $M_{1}$ and $M_{2}$ has an expression similar to (19), it keeps this shape after an arbitrary number of time steps, $A_{0}$ and $B_{0}$ being replaced by $A_{t}$ and $B_{t}$ according to the following recursion

$$
\begin{aligned}
\frac{1}{A_{t+1}} & =2+\frac{1}{A_{t}} \frac{1}{T^{2}} \\
B_{t+1} & =T^{2} B_{t} .
\end{aligned}
$$

This recursion can be solved easily and one finds that

$$
\begin{aligned}
\frac{1}{A_{t}} & =2 \frac{1-T^{-2 t}}{1-T^{-2}}+\frac{T^{-2 t}}{A_{0}} \\
B_{t} & =T^{2 t} B_{0} .
\end{aligned}
$$

From the expressions (23), we see that in the low temperature phase $(T<1), A_{t}$ and $B_{t}$ decrease exponentially with time indicating that the distribution of $M_{1}$ and $M_{2}$ becomes broader and broader. Physically the magnetizations are growing because the system is in its low temperature phase and the probability that $M_{1}$ and $M_{2}$ are small decreases with time.

For $t$ large, each configuration has chosen the valley positive and negative magnetization) it will fall into and the probability $\mathfrak{T}$ that the two configurations fall into different valleys is given by

$$
\mathfrak{T}=\lim _{t \rightarrow \infty} \int_{0}^{\infty} \mathrm{d} M_{1} \int_{-\infty}^{0} \mathrm{~d} M_{2} Q_{t}\left(M_{1}, M_{2}\right)
$$

i.e. by the probability that both configurations have opposite magnetizations. From expressions (A.17) and (A.19) one gets

$$
\begin{aligned}
\mathfrak{T} & =\lim _{t \rightarrow \infty}\left(\frac{1}{2}-\frac{1}{\pi} \tan ^{-1}\left[\frac{B_{t}-A_{t}}{2 \sqrt{A_{t} B_{t}}}\right]\right) \\
& =\lim _{t \rightarrow \infty} \frac{1}{\pi} \tan ^{-1}\left(\frac{2 \sqrt{A_{t} B_{t}}}{B_{t}-A_{t}}\right) \\
& =\lim _{t \rightarrow \infty} \frac{2}{\pi} \tan ^{-1}\left(\sqrt{\frac{A_{t}}{B_{t}}}\right) .
\end{aligned}
$$


From (20), (21) and (23) we see that in the low temperature phase $T<1$, (25) gives for the initial condition (1) :

$$
\mathfrak{T}^{(1)}=\frac{2}{\pi} \tan ^{-1}\left(\sqrt{\frac{1-T^{2}}{T^{2}}}\right)
$$

whereas for initial condition (2), one gets

$$
\mathfrak{T}^{(2)}=\frac{2}{\pi} \tan ^{-1}\left(\sqrt{\frac{1-T^{2}}{1+T^{2}}}\right) .
$$

Figure 1 shows $\mathfrak{T}$ versus $T$ for the two initial conditions (1) and (2). We see that as $T \rightarrow \infty$, $\mathfrak{J}^{(1)} \rightarrow 1$ whereas $\mathfrak{J}^{(2)} \rightarrow 1 / 2$ as expected since at 0 temperature the fact that the same $z_{i}$ are used does not play any role.

As $T$ increases, $T$ decreases because the choice of a valley is less and less influenced by the initial condition and more and more influenced by the effect of thermal noise. These shapes of $\mathfrak{f}^{(1)}$ and $\mathfrak{f}^{(2)}$ are very similar to those which had been observed in numerical simulations of the 3 dimensional Ising model [1].

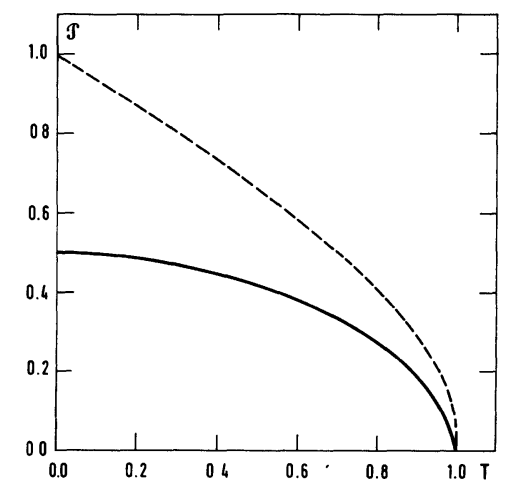

Fig. 1. - Parallel dynamics : probability $\mathfrak{T}$ of finding two configurations submitted to the same thermal noise in different valleys after a large number of time steps as a function of temperature. The dashed curve corresponds to two opposite initial conditions (Eq. (25)) whereas the full curve corresponds to random initial conditions (Eq. (26)).

\section{The time to meet.}

In this section we are going to calculate the time it takes for two configurations to meet once they are in the same valley. We will limit ourselves to the high temperature $(T>1)$ phase because there is a single valley and we will show that the time for two configurations to meet increases like $\log N$. (The same calculation could also be repeated for the case of two configurations which belong to the same valley in the low temperature phase).

If we start with initial conditions (1) or (2) (see section II) at time $t=0$, we have seen in the appendix (Eqs. (A.17, A.19, A.20)) that after time $t$, the distribution $\tilde{Q}(M, D)$ of $M$ and $D$ is given by

$$
\begin{aligned}
\tilde{Q}_{t}(M, D)= & \frac{2}{\pi N} \sqrt{A_{t} B_{t}} \times \\
& \times \exp \left(-\frac{A_{t} M^{2}+B_{t} D^{2}}{N}\right) \theta(D)
\end{aligned}
$$

where $A_{t}$ and $B_{t}$ are related to $A_{0}$ and $B_{0}$ by

$$
\begin{aligned}
\frac{1}{A_{t+1}} & =2+\frac{1}{A_{t}} \frac{1}{T^{2}} \\
B_{t+1} & =T^{2} B_{t} .
\end{aligned}
$$

After a long time $t_{0}$ we see that for $T>1$ one has

$$
\begin{aligned}
A_{t_{0}} & \simeq \frac{1}{2}\left(1-\frac{1}{T^{2}}\right) \\
B_{t_{0}} & =T^{2 t_{0}} B_{0} .
\end{aligned}
$$

So the distribution of $M$ becomes time independent whereas the distribution of the distance becomes narrower and narrower.

From (29b) it is clear that after a time $t_{0}$ of order $\log N, B_{0}$ becomes of order $1 / N$ and the distribution of $D$ becomes concentrated around $D$ of order 1 .

If one defines $\tilde{H}(x, y)$ in the same way as in the appendix,

$$
\tilde{H}_{t}(x, y)=\left\langle\exp \left(\frac{x}{N} M+y D\right)\right\rangle_{t} .
$$

We see from (27) and (29) that after a long time $t_{0}$

$$
\begin{aligned}
\tilde{H}_{t_{0}}(x, y) & =\sqrt{\frac{4}{\pi N} B_{0} T^{2 t_{0}}} \exp \left(\frac{x^{2}}{4 A_{t_{0}} N}\right) \times \\
& \times \int_{0}^{\infty} \mathrm{d} D \exp \left(y D-\frac{B_{0} T^{2 t_{0}} D^{2}}{N}\right) .
\end{aligned}
$$

When the time $t_{0}$ becomes of order $\log N, D$ becomes of order 1 and one can use the results (A.28) and (A.29) of the appendix

$$
\begin{aligned}
\tilde{H}_{t_{0}+\tau}(x, y) & =\sqrt{\frac{4 B_{0} T^{2 t_{0}}}{\pi N}} \exp \left(\frac{x^{2}}{4 A_{t_{0}} N}\right) \\
& \times \int_{0}^{\infty} \mathrm{d} D \exp \left(y_{\tau} D-\frac{B_{0} T^{2 t_{0}} D^{2}}{N}\right) \\
& =\exp \left(\frac{x^{2}}{4 A_{t_{0}} N}\right) E\left[y_{\tau} \sqrt{\frac{N}{B_{0} T^{2 t_{0}}}}\right]
\end{aligned}
$$

where $E(z)$ is an error function

$$
E(z)=\sqrt{\frac{4}{\pi}} \int_{0}^{\infty} \mathrm{d} x \exp \left[z x-x^{2}\right]
$$


where $y_{\tau}$ is related to $y=y_{0}$ by

$$
y_{\tau}=\left(\mathrm{e}^{y_{\tau-1}}-1\right) \frac{1}{T} \text {. }
$$

From (32) and (A.30) one can calculate $Q_{t_{0}+\tau}(0)$ the probability that the distance is 0 at time $t_{0}+\tau$.

$$
\begin{aligned}
& Q_{t_{0}+\tau}(0)=\tilde{H}_{t_{0}+\tau}(0,-\infty)=\tilde{H}\left(0, z_{\tau}\right)= \\
&=E\left(z_{\tau} \sqrt{\frac{N}{B_{0} T^{2 t_{0}}}}\right)
\end{aligned}
$$

where

$$
\begin{aligned}
& z_{\tau}=\left(\mathrm{e}^{z_{\tau-1}}-1\right) \frac{1}{T} \\
& z_{0}=-\infty
\end{aligned}
$$

One can show that for $t \rightarrow \infty, z_{\tau}$ has the following behavior

$$
z_{\tau} \sim \frac{1}{T^{\tau}} \varphi
$$

where $\varphi$ is a constant which depends on the temperature $(\varphi=-.276$ for $T=1.2 ; \varphi=-.486$ for $T=1.5 ; \varphi=-.655$ for $T=2$.).

So we see that after a long time

$$
Q_{t_{0}+\tau}(0)=E\left(\frac{\varphi \sqrt{N}}{\sqrt{B_{0}} T^{t_{0}+\tau}}\right)
$$

which gives clearly for both initial conditions that the probability for the 2 configurations to meet becomes of order 1 when $t_{0}+\tau \sim \log N$.

A similar calculation could be done below $T_{\mathrm{c}}$ for two configurations in the same valley. Again the result would be that it takes a time of order $\log N$ for them to meet.

\section{Finite size effects. Barrier heights.}

For a finite system, one expects that two configurations will always meet after a finite time. This is because for a finite system there is at each time step a finite probability that the two configurations meet. One can then try to study how this time depends on the size $N$. For $T>1$ or for $T<1$ if the two configurations belong to the same valley we have seen (Sect. 3) that this time increases like $\log N$. But in the low temperature phase, one expects this time to be much longer if the two initial configurations belong initially to different valleys. One then expects this time to increase exponentially with $N$ since one of the two configurations has to jump a free energy barrier which increases like $N$ (because the model is a mean field model).
In order to study this effect, we present the results of Monte Carlo simulations done at $T=0.7$ for 10000 samples. In figure 2 we show $\log \left\langle\tau_{2}\right\rangle$ and $\left\langle\log \tau_{2}\right\rangle$ as a function of the number $N$ of spins where $\tau_{2}$ is the time it takes for two randomly chosen initial configurations to meet (initial condition (2)). The average $\langle>$ denotes the average over the initial condition and the history. We see that $\log \left\langle\tau_{2}\right\rangle$ and $\left\langle\log \tau_{2}\right\rangle$ increase with $N$ as expected since these averages of $\tau_{2}$ are dominated by the events, which correspond to the two configurations falling into two opposite valleys.

To confirm this fact we measured (full line in Fig. 2) the relaxation time $\tau$ of the magnetization by calculating the largest eigenvalue $\lambda=\mathrm{e}^{-1 / \tau}$ below 1 of the Master Equation which describes the evolution of the magnetization

$$
P_{t+1}(M)=\sum_{M^{\prime}} \psi\left(M, M^{\prime}\right) P_{t}\left(M^{\prime}\right)
$$

where $\psi\left(M, M^{\prime}\right)$ is the probability that the total magnetization jumps from $M^{\prime}$ to $M$ during a single time step

$$
\begin{aligned}
& \psi\left(M, M^{\prime}\right)=\sum_{n=\max \left(0, \frac{M^{\prime}+M}{2}\right)}^{\min \left(\frac{N+M^{\prime}}{2}, \frac{N+M}{2}\right)}\left(\begin{array}{c}
\frac{N+M^{\prime}}{2} \\
n
\end{array}\right) \times \\
& \times\left(\begin{array}{l}
\frac{N-M^{\prime}}{2} \\
\frac{N+M}{2}-n
\end{array}\right)\left(\frac{1}{2}+\frac{t_{1}}{2}\right)^{n}\left(\frac{1}{2}-\frac{t_{1}}{2}\right)^{\frac{N+M^{\prime}}{2}-n} \\
& \quad \times\left(\frac{1}{2}+\frac{t_{2}}{2}\right)^{\frac{N+M}{2}-n}\left(\frac{1}{2}-\frac{t_{2}}{2}\right)^{n-\frac{M^{\prime}+M}{2}}
\end{aligned}
$$

where $t_{1}=\tanh \frac{M^{\prime}-1}{N T}$ and $t_{2}=\tanh \frac{M^{\prime}+1}{N T}$.

$\lambda=\mathrm{e}^{-1 / \tau}$ is the largest eigenvalue of the odd subspace

$$
\begin{aligned}
\lambda P(M) & =\sum_{M^{\prime}} \psi\left(M, M^{\prime}\right) P\left(M^{\prime}\right) \\
P(M) & =-P(-M) .
\end{aligned}
$$

We see in figure 2 that $\tau$ increases exponentially with $N$ with the same slope as $\tau_{2}$ because both times are dominated by the activation free energy necessary to jump the barrier between the two valleys.

For comparison, we measured also the time $\tau_{3}$ that among 3 randomly chosen initial configurations, two at least meet. We see in figure 2 that $\left\langle\tau_{3}\right\rangle$ does not increase exponentially with $N$. This is of course easy to understand: since below $T_{\mathrm{c}}=1$ there are only 2 valleys, 3 configurations cannot remain different for very long.

Although the fact that $\tau_{2}$ and $\tau_{3}$ have very 


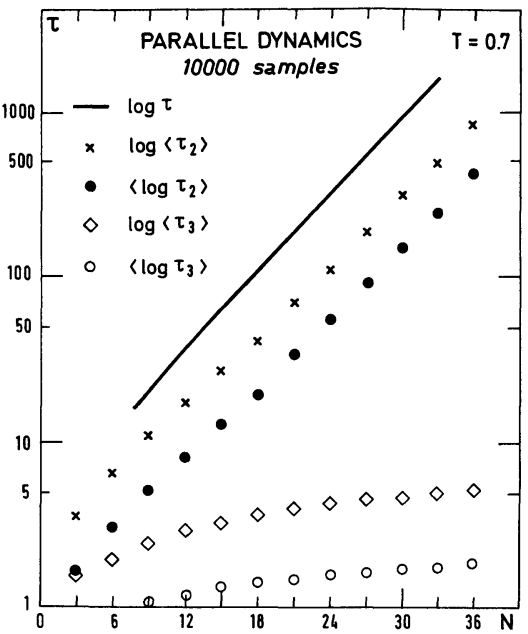

Fig. 2. - Parallel dynamics : in the ferromagnetic phase $\left(T=0.7<T_{\mathrm{c}}=1\right)$, the time $\tau_{2}$ for two configurations to meet as a function of $N$. This time increases exponentially with $N$ as the relaxation time of the magnetization. The slope of $\tau_{2}$ is a measure of the barrier height. The time $\tau_{3}$ that three configurations remain different is much shorter because there are only two valleys.

different behaviors for the mean field ferromagnet is obvious, we think that the same idea could be used to study the barrier heights and the number of valleys in other problems like spin glasses or automata for which the valley structure is expected to be more complex.

\section{Sequential dynamics.}

All the results which have been discussed so far for the case of parallel dynamics can be extended to the case of sequential dynamics : during a time step $\Delta t=\frac{1}{N}$, one chooses a spin $i$ at random among the $N$ spins and this spin $i$ is updated according to (3), (4) and (5). The calculations are very similar to those of parallel dynamics. They are just a little more complicated because condition (A.1) is not fulfilled.

We will not repeat these calculations here and we will just give the final result. For initial conditions (1) and (2), the probability that the two configurations fall into opposite valleys is given by

$$
\begin{aligned}
& \mathfrak{T}^{(1)}=\frac{2}{\pi} \tan ^{-1} \sqrt{2 \frac{1-T}{T}} \\
& \mathfrak{T}^{(2)}=\frac{2}{\pi} \tan ^{-1} \sqrt{1-T} .
\end{aligned}
$$

The shape shown in figure 3 is very similar to what had been obtained in the case of parallel dynamics. $\mathfrak{T}$ decreases with temperature and vanishes at $T_{\mathrm{c}}$.

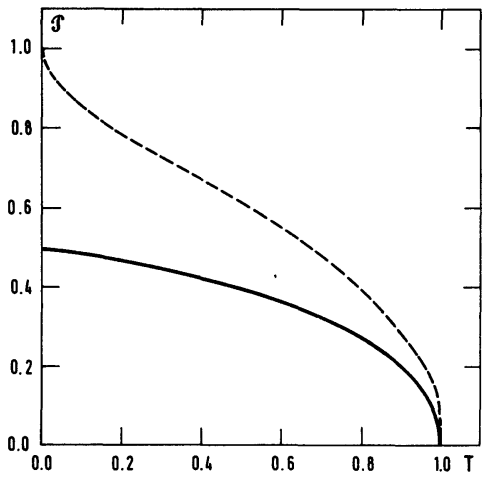

Fig. 3. - Sequential dynamics : survival probability as a function of temperature (as in Fig. 1).

Like for parallel dynamics (Sect. 3) one can also show that the typical time needed for 2 configurations to meet increases like $\log N$ when they are in the same valley.

Lastly, we repeated the simulation of section 4 for sequential dynamics. The results are very similar: $\tau_{2}$ increases exponentially with $N$ and the slope is again the same as for the time $\tau$ (full curve) obtained by diagonalizing the Master equation. Again the time $\tau_{3}$ that 3 configurations remain different is much shorter since the system has only two valleys.

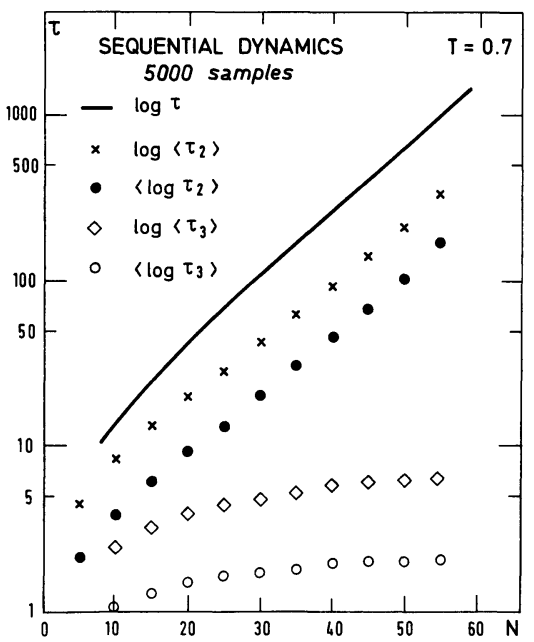

Fig. 4. - Sequential dynamics: the same as figure 2.

\section{Conclusion.}

In this work we have calculated for the mean field ferromagnet the probability that two configurations fall into opposite valleys. This probability decreases with temperature and vanishes at $T_{\mathrm{c}}$ as had been observed in numerical simulations in 3 dimensions [1].

We have also seen that measuring the time that two configurations become identical gives a measure of the barrier heights and that comparing more than 
two configurations can be a method of studying the number of valleys (if a system has $n-1$ valleys, the time $\tau_{n}$ that $n$ different configurations remain different should be very short). Of course for the mean field ferromagnet the results found for $\tau_{2}$ and $\tau_{3}$ are not surprising. However, we think that the study of the times $\tau_{n}$ could be a useful tool [4] to understand the multivalley structure of more complex systems like automata $[5,6]$ or spin glasses [79]. One can also hope that looking at the exponential growth of these times could give a measure of barrier heights [10] in these complex systems.

The results of sections 3 and 4 (the times increase like $\log N$ or an exponential of $N$ ) are valid only for $T \neq T_{\mathrm{c}}$. At $T_{\mathrm{c}}$ one expects that all the times $\tau_{n}$ to be powers of $N$ (for example $\tau_{2} \sim N^{1 / 2}$ ). We tried to calculate for $\tau_{2}$ the prefactor of $N^{1 / 2}$ but we did not succeed in solving the equations analytically.

\section{Acknowledgments.}

We would like to thank V. Hakim and J. L. Lebowitz for useful discussions.

\section{Appendix A}

Suppose that at time $t$, there are $n_{1}$ spins of type I, $n_{2}$ spins of type II, $n_{3}$ spins of type III and $n_{4}$ spins of type IV and that

$$
\begin{array}{cl}
\text { or } & n_{2} \geq n_{3}+1 \\
& n_{3}=0 \\
(i \in \text { type } I & \text { if } \quad S_{i}=\tilde{S}_{i}=+1 \\
i \in \text { type } I I & \text { if } \quad S_{i}=-\tilde{S}_{i}=+1 \\
i \in \text { type } I I I & \text { if } \quad S_{i}=-\tilde{S}_{i}=-1 \\
i \in \text { type } I V & \text { if } \left.\quad S_{i}=\tilde{S}_{i}=-1\right) .
\end{array}
$$

Each spin of type $\mathrm{I}$ at time $t$ has a probability $\frac{1}{2}+\frac{1}{2} t_{1}$ of remaining of type $\mathrm{I}, \frac{1}{2} t_{1}^{\prime}-\frac{1}{2} t_{1}$ of becoming of type II and $\frac{1}{2}-\frac{1}{2} t_{1}^{\prime}$ of becoming of type IV at time $t+1$ where

$$
\begin{aligned}
& t_{1}=\tanh \left(\frac{n_{1}-n_{2}+n_{3}-n_{4}-1}{T N}\right) \\
& t_{1}^{\prime}=\tanh \left(\frac{n_{1}+n_{2}-n_{3}-n_{4}-1}{T N}\right) .
\end{aligned}
$$

Each spin of type II at time $t$ has a probability $\frac{1}{2}+\frac{1}{2} t_{2}$ of becoming of type $\mathrm{I}, \frac{1}{2} t_{2}^{\prime}-\frac{1}{2} t_{2}$ of remaining of type II and $\frac{1}{2}-\frac{1}{2} t_{2}^{\prime}$ of becoming of type IV at time $t+1$ with

$$
\begin{aligned}
& t_{2}=\tanh \left(\frac{n_{1}-n_{2}+n_{3}-n_{4}+1}{T N}\right) \\
& t_{2}^{\prime}=\tanh \left(\frac{n_{1}+n_{2}-n_{3}-n_{4}-1}{T N}\right) .
\end{aligned}
$$

and so on for spins of type III and IV with

$$
\begin{aligned}
& t_{3}=\tanh \left(\frac{n_{1}-n_{2}+n_{3}-n_{4}-1}{T N}\right) \\
& t_{3}^{\prime}=\tanh \left(\frac{n_{1}+n_{2}-n_{3}-n_{4}+1}{T N}\right) \\
& t_{4}=\tanh \left(\frac{n_{1}-n_{2}+n_{3}-n_{4}+1}{T N}\right) \\
& t_{4}^{\prime}=\tanh \left(\frac{n_{1}+n_{2}-n_{3}-n_{4}+1}{T N}\right) .
\end{aligned}
$$

One should notice that as long as condition (A.1) is satisfied, one has $t_{i}^{\prime} \geq t_{i}$ for all $i$ and therefore the number of spins of type III at time $t+1$ is zero.

If $M_{1}$ and $M_{2}$ denote the total magnetization of the configuration $\left\{S_{i}\right\}$ and $\left\{\tilde{S}_{i}\right\}$ and $D$ the distance between these two configurations, $M_{1}, M_{2}$ and $D$ are related to $n_{1}, n_{2}, n_{3}, n_{4}$ by

$$
\begin{aligned}
N & =n_{1}+n_{2}+n_{3}+n_{4} \\
M_{1} & =n_{1}+n_{2}-n_{3}-n_{4} \\
M_{2} & =n_{1}-n_{2}+n_{3}-n_{4} \\
D & =n_{2}+n_{3} .
\end{aligned}
$$

If condition (A.1) is satisfied at $t=0$ (and we will always be in situations where (A.1) is true with probability 1$), n_{3}=0$ at all later times and therefore $M_{1}, M_{2}$ and $D$ are given by

$$
\begin{aligned}
N & =n_{1}+n_{2}+n_{4} \\
M_{1} & =n_{1}+n_{2}-n_{4} \\
M_{2} & =n_{1}-n_{2}-n_{4} \\
D & =n_{2}=\left(M_{1}-M_{2}\right) / 2 .
\end{aligned}
$$

So we see that the system can be fully described by only two variables, for example $M_{1}$ and $M_{2}$.

If we consider the generating function of $M_{1}$ and $M_{2}$ at time $t+1$, it is given by

$$
\begin{aligned}
&\langle\exp (\left.\left.\alpha_{1} M_{1}+\alpha_{2} M_{2}\right)\right\rangle_{t+1}= \\
&=\left\langle\prod _ { i = 1 } ^ { 4 } \left[\cosh \left(\alpha_{1}+\alpha_{2}\right)+t_{i} \mathrm{e}^{\alpha_{1}} \sinh \alpha_{2}\right.\right. \\
&\left.\left.\quad+t_{i}^{\prime} \mathrm{e}^{-\alpha_{2}} \sinh \alpha_{1}\right]^{n_{i}}\right\rangle_{t} .
\end{aligned}
$$

This formula is valid for any distribution of $n_{i}$ at time $t$ which satisfies condition (A.1) and for any $N$.

If condition (A.1) is satisfied, we know that after the first time step $n_{3}=0$ and from (A.7) one can 
calculate the $n_{i}$ and therefore the $t_{i}$ at time $t$. So (A.8) contains the whole time evolution of the probability distribution $Q_{t}\left(M_{1}, M_{2}\right)$ of $M_{1}$ and $M_{2}$. It is however too complicated to allow one to obtain a simple expression of $Q_{t}\left(M_{1}, M_{2}\right)$ after an arbitrary number of time steps because the average \langle\rangle$_{t}$ of the right hand side of (A.9) over $M_{1}$ and $M_{2}$ at time $t$ cannot be expressed in a simple way in terms of the generating function $\left\langle\exp \left(\alpha_{1} M_{1}+\right.\right.$ $\left.\left.\alpha_{2} M_{2}\right)\right\rangle_{t}$. Fortunately there are a few situations for which the calculation of $Q_{t}\left(M_{1}, M_{2}\right)$ has a simple form. We will consider here two of them : Firstly when $M_{1}$ and $M_{2}$ are of order $\sqrt{N}$ which is the case after any finite number of time steps when one starts with random initial conditions. Secondly when $M_{1}$ and $M_{2}$ are both close to one of their equilibrium values.

Let us first discuss the case when $M_{1}$ and $M_{2}$ are of order $\sqrt{N}$. To describe the distribution of $M_{1}$ and $M_{2} \simeq \sqrt{N}$ one can study the time evolution of the generating function $\left\langle\exp \left(\alpha_{1} M_{1}+\alpha_{2} M_{2}\right)\right\rangle_{t}$ with

$$
\alpha_{1}=\frac{x_{1}}{\sqrt{N}} ; \quad \alpha_{2}=\frac{x_{2}}{\sqrt{N}} .
$$

If one defines $H_{t}\left(x_{1}, x_{2}\right)$ by

$$
H_{t}\left(x_{1}, x_{2}\right)=\left\langle\exp \left(\frac{x_{1}}{\sqrt{N}} M_{1}+\frac{x_{2}}{\sqrt{N}} M_{2}\right)\right\rangle_{t} .
$$

One gets from (A.8) for the leading order when $N \rightarrow \infty$

$H_{t+1}\left(x_{1}, x_{2}\right)=\exp \left[\frac{1}{2}\left(x_{1}+x_{2}\right)^{2}\right] H_{t}\left(\frac{x_{1}}{T}, \frac{x_{2}}{T}\right)$.

This recursion relation allows one to calculate the generating function $H_{t}\left(x_{1}, x_{2}\right)$ after any finite number of time steps (to obtain (A.11) from (A.8), we have neglected terms of order $\frac{1}{\sqrt{N}}$ which contribute only on time scales which would increase with $N$ ). From the recursion relation (A.11) on the generating function $H_{t}\left(x_{1}, x_{2}\right)$, one can deduce the probability distribution $Q_{t}\left(M_{1}, M_{2}\right)$ of the magnetizations $M_{1}$ and $M_{2}$ of the two configurations.

If one assumes that at time $t$, the distribution $Q_{t}\left(M_{1}, M_{2}\right)$ is a gaussian with parameters $a_{t}$ and $b_{t}$

$$
\begin{aligned}
& Q_{t}\left(M_{1}, M_{2}\right)=\frac{\sqrt{4 a_{t}^{2}-b_{t}^{2}}}{\pi N} \times \\
& \quad \times \exp \left[-\frac{a_{t}\left(M_{1}^{2}+M_{2}^{2}\right)+b_{t} M_{1} M_{2}}{N}\right] \theta\left(M_{1}-M_{2}\right)
\end{aligned}
$$

(where $\theta(x)=1$ for $x>0$ and 0 for $x<0$ ). One can show from (A.11) that at time $t+1, Q_{t+1}\left(M_{1}, M_{2}\right)$ keeps the form (A.12) with renormalized coefficients $a_{t+1}$ and $b_{t+1}$ given by

$$
\begin{aligned}
& 2 a_{t+1}-b_{t+1}=T^{2}\left(2 a_{t}-b_{t}\right) \\
& \frac{1}{2 a_{t+1}+b_{t+1}}=2+\frac{1}{2 a_{t}+b_{t}} \frac{1}{T^{2}} .
\end{aligned}
$$

An easy way to prove (A.13) and (A.14) is to use the following relation

$$
\begin{aligned}
& \int_{-\infty}^{+\infty} \mathrm{d} M_{1} \int_{-\infty}^{M_{1}} \mathrm{~d} M_{2} Q_{t}\left(M_{1}, M_{2}\right) \times \\
& \times \exp \left[\frac{x_{1} M_{1}+x_{2} M_{2}}{\sqrt{N}}\right]=\sqrt{\frac{2 a_{t}-b_{t}}{\pi}} \int_{0}^{\infty} \mathrm{d} z \\
& \times \exp \left[-z^{2} \frac{\left(2 a_{t}-b_{t}\right)}{4}+\frac{x_{1}-x_{2}}{2} z+\frac{\left(x_{1}+x_{2}\right)^{2}}{4\left(2 a_{t}+b_{t}\right)}\right] .
\end{aligned}
$$

(A.12) can be rewritten as

$$
\begin{aligned}
& Q_{t}\left(M_{1}, M_{2}\right)=\frac{\sqrt{A_{t} B_{t}}}{\pi N} \times \\
& \quad \times \exp \left(-\frac{A_{t}\left(M_{1}+M_{2}\right)^{2}+B_{t}\left(M_{1}-M_{2}\right)^{2}}{4 N}\right) \\
& \quad \times \theta\left(M_{1}-M_{2}\right)
\end{aligned}
$$

where

$$
\begin{aligned}
& A_{t}=2 a_{t}+b_{t} \\
& B_{t}=2 a_{t}-b_{t}
\end{aligned}
$$

and the recursion relations (A.13) and (A.14) become

$$
\begin{aligned}
& \frac{1}{A_{t+1}}=2+\frac{1}{A_{t}} \frac{1}{T^{2}} \\
& B_{t+1}=B_{t} T^{2} .
\end{aligned}
$$

From (A.16), one can deduce the probability distribution $\tilde{Q}_{t}(M, D)$ where $M$ and $D$ are defined by

$$
\begin{gathered}
M=\frac{M_{1}+M_{2}}{2} \\
D=\frac{M_{1}-M_{2}}{2} \\
\tilde{Q}_{t}(M, D)=\frac{2}{\pi N} \sqrt{A_{t} B_{t}} \times \\
\times \exp \left(-\frac{A_{t} M^{2}+B_{t} D^{2}}{N}\right) \theta(D) .
\end{gathered}
$$

These expressions will be used in section 2 .

The second simple situation we want to discuss is when $M_{1}$ and $M_{2}$ are both close to one of their 
equilibrium values and when the distance $D$ is of order 1 i.e. the 2 configurations differ by only a finite number of spins. One can again use (A.8) to obtain the probability distribution of $M_{1}$ and $M_{2}$ or of $M$ and $D$ defined in (A.19). If one defines the generating function $\tilde{H}_{t}(x, y)$ by

$$
\tilde{H}_{t}(x, y)=\left\langle\exp \frac{x}{N} M+y D\right\rangle_{t} .
$$

One can obtain a recursion relation for $\tilde{H}$ from (A.8) by choosing

$$
\alpha_{1}=\frac{y}{2}+\frac{x}{2 N} ; \quad \alpha_{2}=-\frac{y}{2}+\frac{x}{2 N} .
$$

The leading order when $N \rightarrow \infty$ gives

$$
\begin{aligned}
\tilde{H}_{t+1}(x, y)= & \left\langle\operatorname { e x p } \left( x \tanh \left(\frac{M}{T N}\right)+\right.\right. \\
& \left.\left.+\frac{e^{y}-1}{T}\left(1-\tanh ^{2}\left(\frac{M}{T N}\right)\right) D\right)\right\rangle
\end{aligned}
$$

where in (A.23) \langle\rangle means the average over the probability distribution of $M$ and $D$ at time $t$.

If we restrict ourselves to situations where $M$ has an equilibrium value, i.e. is solution of

$$
m=\frac{M}{N}=\tanh \left(\frac{M}{T N}\right)+O\left(\frac{1}{\sqrt{N}}\right)
$$

then (A.22) becomes

$$
\tilde{H}_{t+1}(x, y)=\tilde{H}_{t}\left(x, \frac{\mathrm{e}^{y}-1}{T}\left(1-m^{2}\right)\right) .
$$

We see from (A.25) that the distribution of $\frac{M}{N}$ does not change after one step or any finite number of time steps. This is because we have chosen $M / N$ to be an equilibrium value (A.24).

Since $M / N$ remains fixed, we need only to describe the probability distribution $Q_{t}(D)$ of the distance $D$. The recursion relation (A.25) for the generating function $\tilde{H}_{t}(x, y)$ leads to the following recursion relation for $Q_{t}(D)$ :

$$
\begin{aligned}
Q_{t+1}(D)=\frac{1}{D !} \sum_{D^{\prime}}( & \left.\frac{1-m^{2}}{T} D^{\prime}\right)^{D} \times \\
& \times \mathrm{e}^{-\left(\frac{1-m^{2}}{T} D^{\prime}\right)} Q_{t}\left(D^{\prime}\right)
\end{aligned}
$$

Another consequence of (A.25) is that if at $t=0$, the distance $D$ has a given value $D_{0}$, one has

$$
\tilde{H}_{0}(x, y)=\mathrm{e}^{x \frac{M}{N}} \mathrm{e}^{y D_{0}}
$$

and

$$
\tilde{H}_{t}(x, y)=H_{0}\left(x, y_{t}\right)
$$

where $y_{t}$ can be calculated from $y$ by the following recursion

$$
y_{t}=\left(\mathrm{e}^{y_{t-1}}-1\right) \frac{1-m^{2}}{T}
$$

with $y_{0}=y$.

By choosing $y=-\infty$, one can obtain the probability $Q_{t}(0)$ that the distance $D=0$ at time $t$ since

$$
Q_{t}(0)=\tilde{H}_{t}(0,-\infty)
$$

\section{References}

[1] Derrida, B., Weisbuch, G., Europhys. Lett. 4 (1987) 657.

[2] Barber, M. N., Derrida, B., J. Stat. Phys. 51 (1988) 877.

[3] Fontanari, J. F., Köberle, R., Phys. Rev. A 36 (1987) 2475.

[4] Neumann, A. U., Derrida, B., J. Phys. France 49 (1988) 1647.

[5] de Arcangelis, L., Coniglio, A., preprint (1988).
[6] Derrida, B., Flyvbjerg, H., J. Phys. A 19 (1986) L 1003 ; J. Phys. France 48 (1987) 971.

[7] Gutrreund, H., J. Reger, P. Young, J. Phys. A 21 (1988) 2775.

[8] Sourlas, N., Europhys. Lett. 6 (1988) 561.

[9] Mezard, M., Parisi, G., Sourlas, N., Toulouse, G., Virasoro, M., Phys. Rev. Lett. 52 (1984) 1146 ; J. Phys. France 45 (1984) 843.

[10] Bray, A. J., Moore, M. A., J. Phys. C 17 (1984) L 463 ; Phys. Rev. B 29 (1985) 340. 